\title{
Cesarean Section Among all Deliveries in a Tertiary Care Centre of Nepal: A Descriptive Cross-sectional Study
}

\author{
Renuka Tamrakar, ${ }^{1}$ Sachin Sapkota, ${ }^{1}$ Deekshanta Sitaula, ${ }^{1}$ Rohit Thapa, ${ }^{1}$ Bandana Pokharel,' \\ Suchita Acharya, ${ }^{1}$ Aakriti Parajuli ${ }^{1}$ \\ 'Department of Obstetrics and Gynaecology, Chitwan Medical College, Bharatpur, Nepal.
}

\begin{abstract}
Introduction: Worldwide there is a tremendous increase in cesarean section rate over the last decades which has been a global public health issue. This study aimed to find out the prevalence of cesarean delivery in a tertiary care center of Nepal.
\end{abstract}

Methods: A descriptive cross-sectional study was conducted among pregnant women at tertiary care centre from 15th September 2019 to 15th October 2020. Ethical clearance was taken from the Institutional Review Committee (Ref: CMC-IRC/077/078-200). Convenience sampling was done to reach the sample size. Basic demographic data, clinical indications and neonatal outcomes were noted. Data entry was done using Statistical Package for the Social Sciences version 20. Point estimate at $95 \%$ Confidence Interval was calculated along with frequency and proportion for binary data.

Results: Out of 3193 total deliveries, cesarean deliveries were 1412 (44.22\%) at 95\% Confidence Interval (42.49-45.94). Among caesarean deliveries 1086 (76.9\%) were emergency cesarean sections. Most common indication for cesarean section was fetal distress (24.9\%). Among 1437 newborns, 1428 (99.4\%) were live births, 1387 (98.2\%) were singleton and 801 (55.7\%) were male. Nearly one third $418(29.1 \%)$ neonates required neonatal intensive care unit admission and transient tachypnoea of newborns (44.28\% in emergency and $60.46 \%$ in elective cesarean delivery) was the most common indication for admission.

Conclusions: The prevalence of cesarean delivery was found to be higher than that recommended by the World Health Organisation. Fetal distress was the leading indication for cesarean deliveries.

Keywords: cesarean section; emergency; indications; Nepal.

\section{INTRODUCTION}

Cesarean section (CS) was introduced in late Nineteenth century as a major obstetric intervention to address life-threatening pregnancy and child-birth related complications. ${ }^{1-2}$ It is defined as the birth of foetus through incision in the abdominal wall (laparotomy) and the uterine wall (hysterotomy). ${ }^{1}$

Previous studies from Nepal have reported great variation in rates of CS ranging from $9.5 \%$ to $63 \%$. $^{3-}$ ${ }^{5}$ Although the World Health Organization (WHO) has recommended Cesarean Section rate between 10-15\% for optimal impact, there has been tremendous increase in its rate worldwide over the last decades. ${ }^{6}$ When medically indicated, CS saves lives of both mother and baby, however, unnecessary C-section may adversely affect maternal, neonatal and infant morbidity and mortality. ${ }^{2-3}$

This study aims to find out the prevalence of Cesarean Delivery in a tertiary care center of Nepal.

\section{METHODS}

A descriptive cross-sectional study was conducted among pregnant women who delivered by Cesarean Section at Chitwan Medical College (CMC) from $15^{\text {th }}$ September 2019 to 15th October 2020. Ethical clearance

Correspondence: Dr. Sachin Sapkota, Department of Obstetrics and Gynecology, Chitwan Medical College, Bharatpur, Nepal. Email: hinsac12@gmail.com, Phone: +977-9845595267. 
was taken from the Institutional Review Committee of CMC (Ref: CMC-IRC/077/078-200).

All women who delivered in the centre during the period were included in the study. Informed verbal consent was taken from all the pregnant women. Convenience sampling technique was used and the sample size was calculated using the formula:

$$
\begin{aligned}
\mathrm{n} & =\mathrm{Z}^{2} \times \mathrm{p} \times \mathrm{q} / \mathrm{e}^{2} \\
& =1.96^{2} \times 0.344 \times 0.656 /(0.02)^{2} \\
& =2167.28 \\
& =2168
\end{aligned}
$$

Where,

$\mathrm{n}=$ minimum required sample size

$Z=1.96$ at $95 \%$ confidence interval

$p=$ prevalence reported by a recent study, $34.4 \%^{7}$

$q=1-p$

$\mathrm{e}=$ margin of error, $2 \%$

Considering non-respondent rate of $10 \%$, total sample size becomes 2385. However, our study included 3193 cases of total deliveries.

We recorded socio-demographic information, obstetric background, significant antenatal events, modes of deliveries and obstetric outcomes in a detailed proforma.

Statistical Package for the Social Sciences (SPSS) version 20 was used for data entry and analysis. Point estimate at 95\% Confidence Interval and descriptive statistics were used. Categorical variables were expressed as frequencies whereas continuous variables were expressed as mean \pm SD or median.

\section{RESULTS}

Out of 3193 pregnant women who delivered at Chitwan Medical College during the study period, $1412(44.22 \%)$ at 95\% Confidence Interval (42.49-45.94) women delivered their baby by Cesarean section. The mean age of the women who delivered by CS was $26.44 \pm 4.82$ years (Range 16-49 years). Among cesarean deliveries, more than half of them were multigravida $752(53.26 \%)$ and most of the deliveries were term deliveries 1228 (87\%). Three hundred nineteen (22.6\%) women had underlying comorbidities which included Pregnancy Induced Hypertension (PIH) (38.55\%), hypothyroidism (15.68\%), obstetric cholestasis (13.80\%) and Gestational Diabetes Mellitus (GDM) (13.47\%). Out of 1412 Cesarean deliveries, more than three-fourths 1086 (76.9\%) were emergency Cesarean sections and majority of them 1096 (77.62\%) were primary Cesarean deliveries (Table 1).
Table 1. Obstetric characteristics of women delivered by cesarean section $(n=1412)$.

\begin{tabular}{|ll|}
\hline Gravida & $\mathbf{n}(\%)$ \\
Primi & $660(46.74)$ \\
Multi & $752(53.26)$ \\
Gestational age & \\
Preterm (<37 weeks) & $184(13)$ \\
Term (37-42) & $1211(85.8)$ \\
Post term (>42 weeks) & $17(1.2)$ \\
Type of delivery & \\
Vaginal delivery & $1781(55.78)$ \\
Cesarean Section & $1412(44.22)$ \\
Type of CS & \\
Emergency CS & $1086(76.9)$ \\
Elective CS & $326(23.1)$ \\
Frequency of CS & \\
Primary & $1096(77.62)$ \\
Repeated & $316(22.38)$ \\
\hline
\end{tabular}

Most common indication for CS was found to be fetal distress 352 (24.9\%) followed by previous LSCS 251 $(17.8 \%)$ and meconium-stained liquor 214 (15.2\%) (Table 2).

\begin{tabular}{|lc|}
\hline $\begin{array}{l}\text { Table 2. Indications for cesarean section in study } \\
\text { population ( } \mathbf{n}=1412)\end{array}$ \\
\hline Indications & $\mathbf{n}(\%)$ \\
Fetal distress & $352(24.9)$ \\
Previous LSCS & $251(17.8)$ \\
MSL (Meconium-stained liquor) & $214(15.2)$ \\
Failed IOL/Non progression of labor & $140(9.9)$ \\
Severe Oligohydramnios & $126(8.9)$ \\
Breech & $91(6.4)$ \\
PIH & $61(4.3)$ \\
Cephalo-Pelvic Disproportion & $56(4.0)$ \\
Twins & $20(1.4)$ \\
Others & $101(7.2)$ \\
\hline
\end{tabular}

Among 1437 newborns, 1428 (99.4\%) were live births, 1387 (98.2\%) were singleton and 801 (55.7\%) were male. Three hundred twenty-three $(22.5 \%)$ neonates had low birth weight (LBW). Average weight of newborns delivered by CS was $2902 \pm 591 \mathrm{gm}$. Most of them had an Apgar score of six or more both within one minute $(1410,98.1 \%)$ and within five minutes (1424, 99.1\%) (Table 3).

Table 3. Neonatal outcomes after cesarean section. $(n=1437)^{*}$

\begin{tabular}{|ll|}
\hline Birth outcomes & $\mathbf{n ~ ( \% )}$ \\
Live Birth & $1428(99.4)$ \\
Still Birth & $9(0.6)$ \\
Sex & \\
Male & $801(55.7)$ \\
Female & $636(44.3)$ \\
\hline
\end{tabular}


Tamrakar et al. Cesarean Section in a Tertiary Care Centre of Nepal: A Descriptive Cross-sectional Study

\begin{tabular}{|ll|}
\hline Fetal Number & \\
Singleton & $1387(98.2)$ \\
Twins & $25(1.8)$ \\
Weight of Newborn & \\
$\geq 2.5 \mathrm{~kg}$ & $1114(77.5)$ \\
$1.5-2.5 \mathrm{~kg}(\mathrm{LBW})$ & $300(20.9)$ \\
$1-1.5 \mathrm{~kg}(\mathrm{VLBW})^{\dagger}$ & $18(1.3)$ \\
$<1 \mathrm{~kg}(\mathrm{ELBW})^{\ddagger}$ & $5(0.3)$ \\
Apgar score at 1 minute & \\
0 & $9(0.6)$ \\
$<6$ & $18(1.3)$ \\
$\geq 6$ & $1410(98.1)$ \\
Apgar score at 5 minutes & \\
0 & $9(0.6)$ \\
$<6$ & $4(0.3)$ \\
$\geq 6$ & $1424(99.1)$ \\
\hline
\end{tabular}

* Including 25 twin deliveries, 'Very low birth weight, ${ }^{\ddagger}$ Extremely low birth weight

Nearly one third $418(29.1 \%)$ newborns delivered by CS required NICU admission. Most of them were delivered through emergency CS 331 (79.2\%). About one-third $331(30 \%)$ of total babies delivered via emergency CS and a quarter 87 (25.9\%) of total babies via elective CS required NICU admission. Most common indications for NICU admission were TTN (44.28\% in emergency CS and $60.46 \%$ in elective CS) followed by Neonatal Sepsis $(25.60 \%$ in emergency and $23.30 \%$ in elective CS), respiratory distress syndrome $(12.66 \%$ in emergency CS and $5.8 \%$ in elective CS) and Meconium aspiration syndrome $(6.32 \%$ in emergency CS and $1.16 \%$ in elective CS (Figure 1).

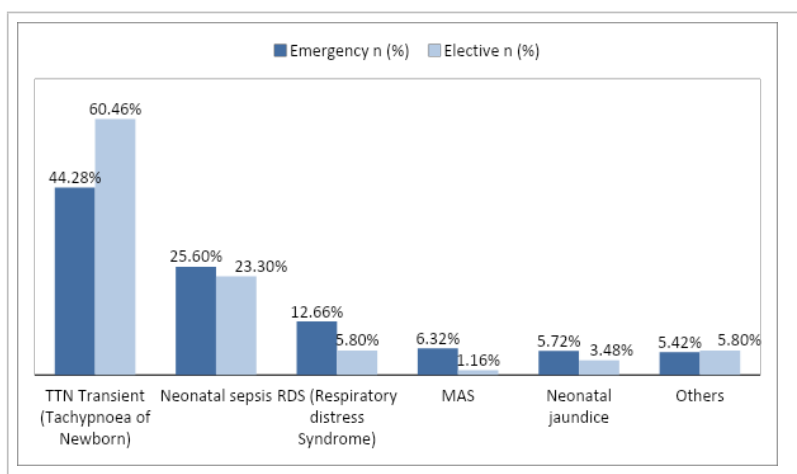

Figure 1. Indications for NICU admission $(n=418)$.

\section{DISCUSSION}

The rate of cesarean section has been in rising trend worldwide with most countries exceeding the WHO recommended rate of $10-15 \% .^{6}$ This rise in CS has been a matter of debate regarding its indications.

Our study shows the CS rate of $44.22 \%$ which is quite towards the higher side as compared to the previous studies carried out in Nepal. Previous studies done in Nepal showed CS rate ranging as high as $63.2 \%$ to as low as $9.5 \%{ }^{4-5}$ Recent study conducted in Kathmandu
Medical College reported similar rate of CS i.e. $45.81 \% .^{8}$ Another study done in Patan academy of health science reported a rise from $38.4 \%$ in 2010 to $46.9 \%$ in 2014 which is nearly similar to our study. ${ }^{9}$ The study done by K.C. et al. reported that the prevalence of CS delivery has increased by four times from 2001 to 2011. ${ }^{10}$ According to WHO, the incidence of CS in USA, England and China is $32 \%, 24 \%$ and $27 \%$ respectively, which is low when compared to our study. ${ }^{11}$ Other Developing countries including India, and regions of South America, also have rates between 25 and $45 \%{ }^{12}$

In 1985, the WHO stated the rate to be not more than $15 \%$ but due to various demographic changes, particularly the increasing maternal age, a target rate of $22 \%$ might be more realistic nowadays. ${ }^{13}$ However, above-mentioned studies including ours has shown even higher rate which is a matter of great concern for all obstetricians. The safe motherhood program by the Government of Nepal may be one of the contributing factors for increasing CS in Nepal as it promotes institutional delivery by providing charge-free delivery. This will somehow affect the decision of mothers as well as clinician for CS delivery. Defensive obstetric practice by clinicians and cesarean delivery on maternal request are also a significant factor for the increase in its rate. However, this may have very little influence in tertiary hospitals like ours because very high-risk cases are usually received for which obstetricians are left with no other choice. Our hospital being situated at the center of the city with advanced facilities and equipment is the choice for many other clinicians from periphery to refer the complicated cases.

The CS rate among primigravida was found to be $46.74 \%$. Pradhan et al. also reported a CS rate of $65.9 \%$ among primigravidae. ${ }^{14}$ These findings are unacceptably high because of implication of CS on the reproductive career for this group of patients.

The leading indication for CS in our study was fetal distress $(24.9 \%)$ which was similar to previous studies from Nepal (19.55\%, 31.5\%).3,8 Pradhan et al. reported a high percentage of fetal distress (40.55\%) as an indication. ${ }^{14}$ Such result could be due to use of cardiotocography as a main indicator for diagnosing fetal distress. It has been reported that cardiotocography monitoring overestimates fetal distress. ${ }^{15-16}$ Estimation of fetal scalp blood $\mathrm{pH}$ is regarded to be gold standard for establishing diagnosis of fetal distress which is not in practice in our institution, not even in other higher tertiary centers over the country. There are range of medical interventions like left lateral positioning, oxygen inhalation to paracervical amnio-infusion for restoration of fetal heart rate. ${ }^{17}$ There is evidence of $70 \%$ success rate with paracervical amnio-infusion. ${ }^{18}$ Therefore, caregiver must be encouraged such practice 
before opting for emergency CS. Overestimation of fetal distress by CTG could be a reason for high CS rate.

Repeat CS (17.8\%), second leading cause for CS explored in this study, is a major contributing factor for global excess of CS rates. American college of obstetrician and gynecologists has clearly instructed that previous CS should not be an indication in absence of any other feto-maternal emergencies. ${ }^{19}$ Many studies claim Vaginal Birth After Cesserian Section (VBAC) to be safer alternatives than repeat CS. ${ }^{12,20}$ RCOG recommended that all women previously delivered by one lower segment CS should be offered an opportunity to labor during their next pregnancy by promoting a trial of scar or of labor..$^{21}$ Medical literature also suggest that $60-80 \%$ of women can safely achieve vaginal delivery. ${ }^{22}$ Rupture of scar was a matter of concern for previous classical cesarean section. However, it became clear that lower segment Cesarean section was not associated with disastrous ruptures. ${ }^{20}$ Regarding our institution, the unwillingness to perform trial of labor after previous CS is probably due to insufficient number of obstetricians as VBAC needs close monitoring, considering $C S$ to be much safer with reduced risk of scar dehiscence or due to maternal preference. Such limited practice of VBAC can be another reason for increased CS rate in our study.

There are few limitations of present study. This is a single-centred study conducted in tertiary level hospitals. So, the findings of this study may not be generalizable. In addition, due to our study design, we were unable to explore the risk factors leading to adverse neonatal outcomes following CS. So, further analytical study is advised to explore those risk factors.

\section{CONCLUSIONS}

The prevalence of cesarean delivery was found to be high in our study. As repeat CS is one of the dominant causes, reduction of primary CS should be given priority. In addition, a comprehensive and evidenced based approach needs to be introduced to monitor the indications of CS and to motivate both provider and recipient for its rational use.

\section{ACKNOWLEDGEMENTS}

We acknowledge the Department of Obstetrics and Gynecology of Chitwan Medical College.

Conflict of Interest: None.

\section{REFERENCES}

1. Teguete I, Traore Y, Sissoko A, Djire M, Thera A, Dolo T, et al. Determining factors of cesarean delivery trends in developing countries: lessons from point $G$ National Hospital (Bamako-Mali) [Internet]. Chine; In Tech. 2012. 200p. Available form: https://www.intechopen.com/ chapters/37219. [Full Text]

2. Begum T, Rahman A, Nababan H, Hoque DME, Khan AF, Ali $\mathrm{T}$, et al. Indications and determinants of Cesarean section delivery: evidence from a population-based study in Matlab, Bangladesh. PloS One. 2017;12(11):e0188074. [ubMed | Full $\underline{\text { Text }}$ | DOI]

3. S Shrestha M, Shrestha S. Cesarean Section profile at a tertiary center. Nepal Journal of Obstetrics and Gynaecology [Internet]. 2020 Jun 7 [cited 28May2021];15(1):68-1. [ Full Text | $\underline{\mathrm{DOI}}]$

4. Samdal LJ, Steinsvik KR, Pun P, Dani P, Roald B, Stray-Pedersen B, et al. Indications for Cesarean Sections in Rural Nepal. J Obstet Gynaecol India. 2016;66(1):284-8. [PubMed $|\underline{\text { Full Text }}| \underline{\text { DOI] }}$

5. Chaudhary R, Raut KB, Pradhan K. Prevalence and indications of cesarean section in a community hospital of western Region of Nepal. JNMA J Nepal Med Assoc. 2018;56(213):871-4. [PubMed | Full Text | DOI]

6. World Health Organisation. Appropriate technology for birth. Lancet. 1985;326(8452):436-7. [PubMed | Full Text | DOI]
7. Shrestha DB, Khatri R, Oli PR, Malla R, Shrestha C, Khatiwada $R$, Silwal P, Shah PB. Cesarean Section in a Maternity Unit of a Tertiary Care Center of Nepal: A Descriptive Cross-sectional Study. JNMA J Nepal Med Assoc [Internet]. 2021 Apr 30 [cited 2021 May 28];59(236):322-6. [Full Text | DOI]

8. Apurwa P, Garima B, Rachana S. Profile of Cesarean Section at Kathmandu Medical College. J Nepal Health Res Counc. 2017;15(2). [uuMed | Full Text | DOI]

9. Pradhan B, Shrestha S, rc L, Sharma P, Bhandary S. Increasing Trend of Cesarean Section in Patan Hospital. Journal of General Practice and Emergency Medicine of Nepal [Internet]. 2015;4(5):2-5. [Full Text]

10. Prakash KC, NeupaneS. Cesarean deliveries among Nepalese mothers: changes over time 2001-2011 and determinants. Arch Gynecol Obstet. 2014;289(2):421-7. [PubMed | Full Text I DOI]

11. Miseljic N, Basic E, Miseljic S. Causes of an Increased Rate of Cesarean Section. Mater Sociomed. 2018;30(4):287-9. [PubMed | Full Text $\mid \underline{\text { DOI] }}$

12. Walker R, Turnbull D, Wilkinson C. Strategies to address global cesarean section rates: a review of the evidence. Birth. 2002;29(1):28-39. [PubMed | Full Text | DOI]

13. van Roosmalen J, van der Does CD. Cesarean birth rates worldwide. A search for determinants. Trop Geogr Med. 1995;47(1):19-22. [ubMed] 
14. Pradhan P, Shrestha S, Rajbhandari P, Dangal G. Profile of Cesarean Section in Kirtipur Hospital [Internet]. Nepal Journal of Obstetrics and Gynaecology. 2014 Dec 16. [cited 2021 May 28];9(2):51-4. [Full Text | DOI]

15. Unnikrishnan B, Rakshith P, Aishwarya A, Nithin K, Rekha T, Prasanna P, et al. Trends and Indications for Caesarean Section in a tertiary care Obstetric Hospital in Coastal South India. Australasian Medical Journal. 2010;3(12):821-5. [Full Text $\mid \underline{\text { DOI] }}$

16. Dabbas M, Al-Sumadi A. Cesarean section rate: much room for reduction. Clin Exp Obstet Gynecol. 2007;34(3):146-8. [PubMed | Full Text]

17. Hofmeyr GJ, $\mathrm{Xu}$ H, Eke AC. Amnioinfusion for meconium-stained liquor in labour. Cochrane Database Syst Rev. 2010;(1):CD000014. [uued | Full Text | DOI]

18. Abdel-Aleem H, Amin A, Shokry M, Radwan R. Therapeutic amnioinfusion for intrapartum fetal distress using a pediatric feeding tube. Int J Gynaecol Obstet. 2005;90(2):94-8. [PubMed | Full Text | DOI]
19. Obstetricians ACo, Gynecologists. Vaginal birth after previous cesarean delivery. Obstet Gynecol. 2010;116(2 Pt 1):450-63. [ubMed | Full Text | DOI]

20. Penn Z, Ghaem-Maghami S. Indications for caesarean section. Best Pract Res Clin Obstet Gynaecol. 2001;15(1):1-15. [PubMed | Full Text | DOI]

21. Thomas J, Paranjothy S. National Sentinel Caesarean Section Audit Report. London: RCOG press; 2001. 117p. Available from: https://www.rcog.org.uk/globalassets/documents/ guidelines/research--audit/nscs_audit.pdf [ Full Text]

22. Sharma A, Acharya R, Sharma U, Chaudhary A, Chaudhary P, Hanspal J. Maternal and Neonatal Outcome in Patients with History of Previous One Cesarean Section. Indian Medical Gazette. 2012 May;145(5):169-73. [Full Text]

\section{The Author(s) 2018.}

This work is licensed under a Creative Commons Attribution 4.0 International License. The images or other third party material in this article are included in the article's Creative Commons license, unless indicated otherwise in the credit line; if the material is not included under the Creative Commons license, users will need to obtain permission from the license holder to reproduce the material. To view a copy of this license, visit http://creativecommons.org/licenses/by/4.0/ 